\title{
GAMIFICAÇÃO NO ENSINO SUPERIOR: UMA ANÁLISE DE ESTUDOS ACADÊMICOS REALIZADOS EM PORTUGAL E NO BRASIL
}

\author{
Gamification in Higher Education: an Analysis of Academic Studies Carried out in \\ Portugal and Brazil
}

\author{
Glaucia Diniz Marques ${ }^{1}$ \\ Fernando Albuquerque Costa ${ }^{2}$
}

\begin{abstract}
Resumo: Em resposta ao problema da desmotivação dos estudantes face ao ensino uniforme e massivo a que são sujeitos no contexto do ensino superior, são cada vez mais frequentes as experiências de aprendizagem alternativas, promovidas pelos docentes, com o objetivo de aumentar o interesse e envolvimento na aprendizagem. Uma dessas linhas de trabalho alternativas com forte implantação na última década é a gamificação que, como o próprio nome sugere, visa trazer uma dimensão lúdica para o processo de ensino e de aprendizagem para, dessa forma, aproveitando a adesão natural dos jovens à experiência de jogo, conseguir também melhores resultados em termos do seu desempenho e rendimento escolar. Uma vez que nos interessa conhecer o que nesse âmbito está sendo realizado no contexto da língua portuguesa, apresentamos um estudo sobre os trabalhos acadêmicos (dissertações de mestrado e teses de doutoramento) desenvolvidos nos últimos cinco anos na temática da gamificação da aprendizagem no contexto do ensino superior, em Portugal e no Brasil. Tendo como base uma revisão sistemática da literatura, o objetivo foi o de fazer uma primeira aproximação do que vem sendo estudado nos dois países em resposta às questões que vêm sendo pesquisadas na área das Ciências Sociais e Humanas, sobre os modelos teóricos de enquadramento, sobre as principais metodologias utilizadas, e os principais resultados e conclusões a que esses estudos chegaram. Os resultados mostram que todas as investigações selecionadas fazem em termos gerais uma avaliação positiva do recurso à gamificação (aumento da motivação, aprendizagem, interação, gamificação como inovação pedagógica).
\end{abstract}

Palavras-chave: Gamificação. Ensino Superior. Revisão Sistemática.

Abstract: In response to the problem of students' demotivation in the face of uniform and massive teaching to which they are subjected in the context of higher education, alternative learning experiences, promoted by teachers, are increasingly frequent, with the aim of increasing interest and involvement in learning. One of these alternative lines of work with a

\footnotetext{
${ }^{1}$ Doutoranda em Educação no Instituto de Educação da Universidade de Lisboa. Mestre em Educação pela Universidade do Estado do Rio de Janeiro (UERJ). Professora na Universidade Estácio de Sá. Orcid: https://orcid.org/0000-0003-0181-3196. Email: glauciadm@gmail.com

2 Professor Auxiliar e investigador de Tecnologia da Educação no Instituto de Educação da Universidade de L Lisboa. É o diretor acadêmico do Mestrado em Educação e Formação (e-Learning e Ensino à Distância) e do curso de Educação e Formação. Atualmente, participa no projeto Game Course - Improving College Learning with Gamification, um projeto financiado pela FCT. Orcid: https://orcid.org/0000-0001-9604-5542. Email: fc@ie.ulisboa.pt
} 
strong implantation in the last decade is gamification, which (as its name suggests) aims to bring a playful dimension to the teaching and learning process, thus taking advantage of the natural adhesion of young people to the experience game, also achieving better results in terms of their school performance and development. Since we are interested in knowing what is being done in this context in considering the Portuguese language sphere, we present a study on the academic works (master's dissertations and doctoral theses) developed in the last five years on the theme of gamification of learning in the context of teaching higher education in Portugal and Brazil. Based on a systematic review of the literature, the objective was to make a first approximation of what has been studied in these two countries in response to the questions that have been researched in the area of Social and Human Sciences, about the theoretical framework models, about the main methodologies used, and the main results and conclusions reached by these studies. The results show that all selected investigations generally make a positive assessment of the use of gamification (increased motivation, learning, interaction, gamification as a pedagogical innovation).

Keywords: Gamification. University education. Systematic review.

\section{Introdução}

A presença dos jogos no cotidiano dos jovens é um fato consolidado principalmente entre as novas gerações, conhecidas como Geração Y ou Millenials e Geração Z ou iGeneration. Por esse motivo, compreende-se que, na escola, tais gerações exigem novas formas de ensino, com abordagens mais dinâmicas e desafiantes. A gamificação tem aparecido na literatura precisamente como uma alternativa para motivar os estudantes, pois consegue tornar o processo mais interessante e mais dinâmico. Como para esses jovens, a utilização de recursos digitais é uma atividade natural no seu dia a dia, é de se esperar que a educação possa aproveitar essa grande desenvoltura e a competência efetiva no uso de tecnologias para proporcionar situações de ensino e aprendizagem nas quais possam utilizar dispositivos e ambientes digitais que se assemelham aos que usam no seu cotidiano, projetando-os de forma a promover, também, um maior envolvimento nas atividades de aprendizagem e, consequentemente, um melhor desempenho acadêmico. Prensky (2012) refere-se a esse propósito que a aprendizagem baseada em jogos digitais é importante porque atende às necessidades e aos estilos de aprendizagem da geração atual, podendo ser adaptada e utilizada em diferentes disciplinas e, quando usada de forma adequada, ser eficaz, para além de divertida. $\mathrm{Na}$ área dos negócios e também inspirada na cultura dos jogos, há diversos anos que a gamificação tem expandido, com abordagens que envolvem nomeadamente captação e fidelização de clientes às empresas. De acordo com Carvalho (2012), criar jogos com o objetivo de atrair consumidores para as lojas ou motivar os seus funcionários também não é nada novo. A novidade é precisamente a introdução de tecnologia nesse processo, sendo muitos os casos de empresas tecnológicas que entraram no mundo da gamificação como, por exemplo, a Microsoft, a Samsung ou a SAP, com o objetivo de aumentar o envolvimento dos seus funcionários e clientes, recorrendo a conceitos relacionados a jogos em suas tarefas diárias.

O conceito de gamificação é entendido como a adoção de elementos de jogo em contextos fora do jogo (DETERDING et al., 2011; HUOTARI; HAMARI, 2012). Refere-se a atividades transformadoras, sistemas, produtos, serviços ou estruturas organizacionais para proporcionar experiências divertidas (HAMARI, 2019).

No ensino superior, a gamificação surge recorrentemente em reação ao problema da desmotivação, frustração e abandono escolar dos estudantes (OLIVEIRA; BITTENCOURT, 
2019; VIAMONTE, 2018; TENÓRIO et al., 2016). Uma situação devido à forma como o currículo é trabalhado, proporcionando um ensino marcadamente uniforme e estandardizado, um currículo que oferece o mesmo a todos, independentemente da especificidade dos indivíduos a quem é dirigido, das características e ambições pessoais de cada um. A abordagem à aprendizagem pela gamificação surge, desse modo, como uma resposta ao desinteresse dos estudantes pela aprendizagem (MAZUR, 2020; HASSAN et al., 2019), uma vez que tem como propósito tornar a experiência de aprender na universidade muito mais divertida, interessante, apelativa e, por via disso, bem-sucedida.

No que diz respeito à percepção dos estudantes sobre esse tipo de experiências de aprendizagem alternativas, Bai, Hew e Huang (2020) identificam quatro principais ordens de argumentos enunciados pelos estudantes para justificarem o seu apreço pela gamificação. Ao contrário de uma escola monótona e desinteressante, os estudantes reconhecem que a gamificação, de uma forma geral, estimula o seu gosto e entusiasmo pelo que estão aprendendo. Os estudantes destacam, por outro lado e em particular, a utilidade efetiva que reconhecem ao feedback imediato sobre o seu desempenho, na medida em que contribui para a manutenção dos seus índices motivacionais sobre as tarefas a realizar. Sublinham, também, a possibilidade que a gamificação oferece em termos de satisfação da necessidade de reconhecimento. Os estudantes referem, por último, que o fato de estarem envolvidos num processo de ensino e aprendizagem gamificado os estimula a estabelecer as suas próprias metas, o que, conforme suas opiniões, acaba sendo determinante em termos de maior controle sobre o próprio processo e que nas situações de ensino normais, ou seja, não gamificadas, nem sempre acontece.

Aproveitando a adesão natural que os jovens manifestam relativamente à experiência de jogo em geral (pelo prazer, adrenalina e desafio que os proporciona), e, particularmente, o interesse que, do ponto de vista pedagógico, vai sendo relatado na literatura, em escala mundial sobre as experiências de gamificação que os estudantes são envolvidos, pareceu-nos pertinente conhecer o nível em que será realizado, especificamente, no contexto dos dois países (Brasil e Portugal). Nos pontos subsequentes, apresentaremos o estudo que realizamos sobre os trabalhos acadêmicos (dissertações de mestrado e teses de doutoramento) desenvolvidos nos últimos cinco anos na temática da gamificação da aprendizagem no contexto do ensino superior em Portugal e no Brasil.

\section{Metodologia}

\subsection{Natureza do estudo}

Trata-se de uma primeira aproximação ao campo de estudo, ou seja, aos trabalhos realizados na temática da gamificação no ensino superior no contexto dos dois países. Optamos por proceder a uma revisão sistemática da literatura, seguindo um conjunto de procedimentos habituais sugeridos para este tipo de trabalho: propósito do estudo; fontes de dados; construção do corpus de análise; critérios de inclusão e de garantia de qualidade, dentre outros, conforme apresentado nos pontos seguintes.

\section{2 Âmbito e objetivos}

Através de um processo de revisão sistemática, tendo como base teses e dissertações relatando experiências de gamificação no ensino superior, pretendia-se em concreto: a) compreender o que no geral tem sido estudado a propósito da gamificação na área das Ciências Sociais e Humanas (temáticas); b) identificar abordagens, modelos e frameworks tomados 
como referência (teorias); c) identificar quais as principais metodologias utilizadas; e, por último, d) conhecer os principais resultados e conclusões a que esses estudos chegaram.

\subsection{Fontes de dados}

Para a realização do estudo, recorremos à base de dados da produção científica aberta existente em Portugal, conhecida como Portal RCAAP - Repositórios Científicos de Acesso Aberto de Portugal, por se tratar da principal base de dados validados pela comunidade científica e por permitir o acesso aberto a um número significativo de documentos. Apesar de se tratar de um repositório português, desde o ano de 2010, essa base passou a agregar conteúdos científicos do Instituto Brasileiro de Informação em Ciência e Tecnologia (IBICT), o que permitiria aceder também à produção científica realizada no Brasil.

\subsection{Constituição do corpo documental}

Numa primeira etapa da consulta, realizada entre dezembro do ano 2019 e fevereiro de 2020, utilizaram-se os descritores "Gamificação", "Gamificar" e "Gamificado", tendo sido identificados 337 documentos, distribuídos por instituições públicas e privadas de Portugal e do Brasil.

Numa segunda etapa, para se proceder à depuração e seleção final dos documentos que iriam constituir o corpus documental, foi feita a leitura de título, palavras-chave, resumo, considerações finais e, em alguns casos, a introdução tendo como referência os critérios de inclusão e exclusão que constam no Quadro 1 (Critérios de seleção).

Quadro 1 - Critérios de seleção

\begin{tabular}{|l|l|}
\hline \multicolumn{1}{|c|}{ Critérios de Inclusão } & \multicolumn{1}{c|}{ Critérios de Exclusão } \\
\hline $\begin{array}{l}\text { Dissertações de Mestrado e Teses de } \\
\text { Doutorado sobre Gamificação no } \\
\begin{array}{l}\text { Ensino Superior na área das Ciências } \\
\text { Humanas ou Sociais }\end{array}\end{array}$ & $\begin{array}{l}\text { Todo e qualquer tipo de documento que } \\
\text { não fosse tese ou dissertação na área das } \\
\text { Ciências Humanas ou Sociais }\end{array}$ \\
\hline $\begin{array}{l}\text { Publicações no período de janeiro de } \\
2015 \text { a fevereiro de 2020 }\end{array}$ & Publicações anteriores a janeiro de 2015 \\
\hline $\begin{array}{l}\text { Documentos com acesso aberto no } \\
\text { RCAAP }\end{array}$ & $\begin{array}{l}\text { Documentos com acesso embargado ou } \\
\text { restrito }\end{array}$ \\
\hline & Documentos duplicados \\
\hline
\end{tabular}

Fonte: Elaborado pelos autores, 2021.

Da aplicação sucessiva dos referidos critérios, chegou-se, num primeiro momento, a um total de 74 documentos abordando aplicações da gamificação no ensino superior. No entanto, quando aplicados os critérios de exclusão, nomeadamente o critério relativo à área do conhecimento, deparou-se com um número de estudos reduzido a um total de 26 estudos na área das Ciências Sociais e Humanas. A lista dos estudos sobre os quais incidiu a análise é disponibilizada em anexo.

\subsection{Procedimentos de análise}

Em seguida, procedeu-se à análise qualitativa do conteúdo dos textos selecionados, tendo em vista identificar os elementos que permitissem enquadrar cada investigação nos critérios previamente estabelecidos. Para isso, foi construída uma estrutura de análise com base 
nos objetivos mencionados anteriormente, ou seja, visando primeiramente à identificação das temáticas e tópicos tratados, em segundo lugar à caracterização da experiência de gamificação propriamente dita, depois, à identificação das opções metodológicas seguidas nos estudos e, por fim, à identificação dos principais resultados obtidos com as experiências de gamificação.

No que se refere à identificação das problemáticas estudadas, tomamos como referência, inicialmente, a análise das palavras-chave e dos títulos dos trabalhos a fim de melhor compreender o alcance temático dos estudos (IMBELLONI, 2012). Depois, num segundo momento e com o objetivo de melhor compreender qual o foco principal dos estudos efetuados, utilizamos a proposta de Parreño, Ibañez e Arroyo (2016), que sugerem uma taxonomia específica para os estudos de gamificação. Para esses autores, são quatro as dimensões que importa considerar na análise: efetividade, aceitação, envolvimento e interações sociais.

Relativamente à caracterização das experiências de gamificação propriamente dita, tomaram-se em consideração todas as referências explícitas dos autores sobre os principais conceitos e modelos subjacentes à organização do processo de ensino e aprendizagem sujeito à gamificação, ou seja, os princípios que de alguma forma contribuem para a configuração das propostas pedagógicas respectivas, mas também a referência aos elementos do jogo considerados (DETERDING et al., 2011) e às tecnologias utilizadas para a sua concretização.

Para caracterizar a metodologia utilizada nos estudos, a atenção centrou-se principalmente nas referências explícitas sobre a natureza da investigação (pendor qualitativo ou quantitativo, abordagem mista ou metodologia de desenvolvimento) e o tipo de instrumentos prevalecentes em termos de recolha de dados (questionário, entrevista, diário de bordo, grupo focal, observação, etc.).

Por último, com o intuito de compreender os principais resultados obtidos, tomamos como referência as considerações dos autores, quer em função das temáticas mais recorrentes (por exemplo, em termos de inovação pedagógica ou de aprendizagem dos estudantes), quer em função da própria avaliação geral que é feita (balanço satisfatório versus balanço insatisfatório), e também em termos dos limites e desafios apontados, visando futuras pesquisas na área.

\section{Síntese dos principais resultados}

Antes mesmo de se proceder à caracterização dos estudos analisados, importa começar por referir a sua distribuição em termos de país de origem e respectivas datas de publicação. Com esse objetivo e de acordo com o Quadro 2 (ano e origem dos trabalhos), observou-se que o investimento em trabalhos sobre gamificação tem sido bastante maior no contexto do ensino superior brasileiro, com cerca de dois terços do trabalho realizados (19 trabalhos), sendo também evidente o aumento e estabilização do interesse por esse tipo de estudos no âmbito de trabalhos acadêmicos (com 21 trabalhos entre os anos de 2017 e 2019). 
Quadro 2 - Ano e origem dos trabalhos

\begin{tabular}{|l|c|c|c|c|c|c|c|c|}
\hline \multirow{2}{*}{ Autor } & \multicolumn{5}{|c|}{ Ano } & \multicolumn{3}{c|}{ Origem } \\
\hline & 2015 & 2016 & 2017 & 2018 & 2019 & 2020 & Brasil & Portugal \\
\hline (P1) Santos & & & & $\mathrm{X}$ & & & $\mathrm{X}$ & \\
\hline (P2) Quadros & & $\mathrm{X}$ & & & & & $\mathrm{X}$ & \\
\hline (P3) França & & $\mathrm{X}$ & & & & & $\mathrm{X}$ & \\
\hline (P4) Sataka & & & & & $\mathrm{X}$ & & $\mathrm{X}$ & \\
\hline (P5) Corcini & & $\mathrm{X}$ & & & & & $\mathrm{X}$ & \\
\hline (P6) Jacobsen & & & & $\mathrm{X}$ & & & $\mathrm{X}$ & \\
\hline (P7) Rodrigues & & & & $\mathrm{X}$ & & & & $\mathrm{X}$ \\
\hline (P8) Crespo & & & & & $\mathrm{X}$ & & $\mathrm{X}$ & \\
\hline (P9) Guedes & & & $\mathrm{X}$ & & & & $\mathrm{X}$ & \\
\hline (P10) Duarte & & & $\mathrm{X}$ & & & & $\mathrm{X}$ & \\
\hline (P11) Pessi & & & & $\mathrm{X}$ & & & $\mathrm{X}$ & \\
\hline (P12) Tristão & & & & $\mathrm{X}$ & & & $\mathrm{X}$ & \\
\hline (P13) Gomes & & & $\mathrm{X}$ & & & & $\mathrm{X}$ & \\
\hline (P14) Silva & & & & & $\mathrm{X}$ & & & $\mathrm{X}$ \\
\hline (P15) Gomes & & & & $\mathrm{X}$ & & & & $\mathrm{X}$ \\
\hline (P16) Saraiva & & & & $\mathrm{X}$ & & & & $\mathrm{X}$ \\
\hline (P17) Simba & & & $\mathrm{X}$ & & & & & $\mathrm{X}$ \\
\hline (P18) Gervásio & & & & & $\mathrm{X}$ & & $\mathrm{X}$ & \\
\hline (P19) Gomes & & & $\mathrm{X}$ & & & & $\mathrm{X}$ & \\
\hline (P20) Pombo & & & & & $\mathrm{X}$ & & & $\mathrm{X}$ \\
\hline (P21) Ferreira & $\mathrm{X}$ & & & & & & $\mathrm{X}$ & \\
\hline $\begin{array}{l}\text { (P22) } \\
\text { Alexandre }\end{array}$ & & & & & $\mathrm{X}$ & $\mathrm{X}$ & \\
\hline (P23) Sobreiro & & & $\mathrm{X}$ & & & & $\mathrm{X}$ & \\
\hline (P24) Caixado & & & & & $\mathrm{X}$ & & & $\mathrm{X}$ \\
\hline (P25) Quaresma & & & & & $\mathrm{X}$ & & $\mathrm{X}$ & \\
\hline (P26) Coelho & & & $\mathrm{X}$ & & & & $\mathrm{X}$ & \\
\hline \multicolumn{1}{|c|}{ Total (\%) } & $1(4)$ & $3(11)$ & $7(27)$ & $7(27)$ & $7(27)$ & $1(4)$ & $19(73)$ & $7(27)$ \\
\hline
\end{tabular}

\subsection{Identificação das áreas e dimensões estudadas}

Com o intuito de identificar as principais áreas de ensino em que decorreram os trabalhos selecionados, utilizamos, num primeiro momento, uma estratégia de análise tendo como base os títulos dos trabalhos, as palavras-chave e os respectivos resumos. Como observase no Quadro 3 (Áreas de ensino), verifica-se uma grande diversidade de áreas de ensino, sendo a maior parte das experiências de gamificação realizada no contexto de ensino de Línguas estrangeiras, com oito estudos, e no ensino de Matemática e de temas de Educação, ambas com seis estudos cada. 
Quadro 3 - Áreas de ensino

\begin{tabular}{|c|c|c|c|c|c|c|c|c|c|}
\hline \multirow[b]{2}{*}{ Autor } & \multicolumn{9}{|c|}{ Área de ensino } \\
\hline & $\begin{array}{l}\text { Líng } \\
\text { uas }\end{array}$ & $\begin{array}{l}\text { Mate } \\
\text { máti } \\
\text { ca }\end{array}$ & $\begin{array}{l}\text { Hist } \\
\text { ória }\end{array}$ & $\begin{array}{l}\text { Admini } \\
\text { stração }\end{array}$ & $\begin{array}{l}\text { Educa } \\
\text { ção }\end{array}$ & $\begin{array}{c}\text { Saú } \\
\text { de }\end{array}$ & $\begin{array}{c}\text { Marke } \\
\text { ting }\end{array}$ & $\begin{array}{c}\text { Psicol } \\
\text { ogia }\end{array}$ & $\begin{array}{l}\text { Inf } \\
\text { or } \\
\text { mát } \\
\text { ica }\end{array}$ \\
\hline (P1) Santos, 2018 & & & & & $\mathrm{X}$ & & & & \\
\hline (P2) Quadros, 2016 & $\mathrm{X}$ & & & & & & & & \\
\hline (P3) França, 2016 & & & & $\mathrm{X}$ & & & & & \\
\hline (P4) Sataka, 2019 & $\mathrm{X}$ & & & & & & & & \\
\hline (P5) Corcini, 2016 & & & & & $\mathrm{X}$ & & & & \\
\hline (P6) Jacobsen, 2018 & & $\mathrm{X}$ & & & & & & & \\
\hline (P7) Rodrigues, 2018 & & & & & & $\mathrm{X}$ & & & \\
\hline (P8) Crespo, 2019 & $\mathrm{X}$ & & & & & & & & \\
\hline (P9) Guedes, 2017 & & & $\mathrm{X}$ & & & & & & \\
\hline (P10) Duarte, 2017 & $\mathrm{X}$ & & & & & & & & \\
\hline (P11) Pessi, 2018 & & & & & $\mathrm{X}$ & & & & \\
\hline (P12) Tristão, 2018 & & & & & $\mathrm{X}$ & & & & \\
\hline (P13) Gomes, 2017 & & $\mathrm{X}$ & & & & & & & \\
\hline (P14) Silva, 2019 & & & & & & & $\mathrm{X}$ & & \\
\hline (P15) Gomes, 2018 & $\mathrm{X}$ & & & & & & & & \\
\hline (P16) Saraiva, 2018 & & & & & $\mathrm{X}$ & & & & \\
\hline (P17) Simba, 2017 & & & & & & & & $\mathrm{X}$ & \\
\hline (P18) Gervásio, 2019 & $\mathrm{X}$ & & & & & & & & \\
\hline (P19) Gomes, 2017 & & $\mathrm{X}$ & & & & & & & \\
\hline (P20) Pombo, 2019 & $\mathrm{X}$ & & & & & & & & \\
\hline (P21) Ferreira, 2015 & & $\mathrm{X}$ & & & & & & & \\
\hline (P22) Alexandre, 2020 & $\mathrm{X}$ & & & & & & & & \\
\hline (P23) Sobreiro, 2017 & & & & & & & & & $\mathrm{X}$ \\
\hline (P24) Caixado, 2019 & & & & & $\mathrm{X}$ & & & & \\
\hline (P25) Quaresma, 2019 & & $\mathrm{X}$ & & & & & & & \\
\hline (P26) Coelho, 2017 & & $\mathrm{X}$ & & & & & & & \\
\hline Total $(\%)$ & $8(31)$ & $6(23)$ & $1(4)$ & $1(4)$ & $6(23)$ & $1(4)$ & $1(4)$ & $1(4)$ & $1(4)$ \\
\hline
\end{tabular}

Fonte: Elaborado pelos autores, 2021.

No caso da gamificação associada ao ensino das Línguas, e a título de exemplo, destacamos a investigação de Quadros (2016) cujo objetivo é compreender o modo como os elementos da gamificação podem ou não oferecer as condições para que os aprendizes se aproximem do "estado de fluxo" proposto por Csikszentmihalyi (1990) durante o processo de aprendizagem de uma língua estrangeira, em uma ferramenta de autoria de recursos educacionais online.

Num segundo momento, percebermos qual o principal motivo e o foco dos trabalhos, e apoiados na taxonomia proposta por Parreño, Ibañez e Arroyo (2016), foi possível concluir que são principalmente as questões relacionadas ao envolvimento dos estudantes na aprendizagem que preocupam os investigadores, com seis estudos, muito embora a aceitação desse tipo de estratégias alternativas de organização da aprendizagem pelos estudantes e as questões referentes à sua eficácia estejam também presentes de forma distinta, com cinco e quatro estudos respectivamente. A observação do Quadro 4 (Dimensões estudadas) permite também perceber que apenas dois dos estudos analisados incluem em simultâneo as três dimensões anteriormente referidas. Pode-se observar, por outro lado, que a dimensão Interação social, com apenas dois estudos, foi a dimensão que menor atenção dedicaram os investigadores. No quadro, notou-se, ainda, que cerca de metade dos estudos tem como foco outros aspetos, dos 
quais destacamos a perspectiva dos estudantes sobre os elementos do jogo disponibilizados ou sobre as próprias tecnologias utilizadas na criação das experiências de gamificação.

Quadro 4 - Dimensões estudadas

\begin{tabular}{|c|c|c|c|c|c|}
\hline \multirow[b]{2}{*}{ Autor } & \multicolumn{5}{|c|}{ Foco do estudo } \\
\hline & Envolvimento & Aceitação & Efetividade & $\begin{array}{c}\text { Interação } \\
\text { social }\end{array}$ & Outros \\
\hline \multicolumn{6}{|l|}{ (P1) Santos, 2018} \\
\hline (P2) Quadros, 2016 & $\mathrm{X}$ & & & & \\
\hline (P3) França, 2016 & & & & $\mathrm{X}$ & \\
\hline \multicolumn{6}{|l|}{ (P4) Sataka, 2019} \\
\hline (P5) Corcini, 2016 & & & & & $\mathrm{X}$ \\
\hline (P6) Jacobsen, 2018 & & & & & $\mathrm{X}$ \\
\hline (P7) Rodrigues, 2018 & & & $\mathrm{X}$ & & \\
\hline (P8) Crespo, 2019 & $\mathrm{X}$ & & & & $\mathrm{X}$ \\
\hline (P9) Guedes, 2017 & $\mathrm{X}$ & $\mathrm{X}$ & $\mathrm{X}$ & & \\
\hline \multicolumn{6}{|l|}{ (P10) Duarte, 2017} \\
\hline \multicolumn{6}{|l|}{ (P11) Pessi, 2018} \\
\hline (P12) Tristão, 2018 & & & & & $\mathrm{X}$ \\
\hline \multicolumn{6}{|l|}{ (P13) Gomes, 2017} \\
\hline (P14) Silva, 2019 & $\mathrm{X}$ & $\mathrm{X}$ & $\mathrm{X}$ & & $\mathrm{X}$ \\
\hline \multicolumn{6}{|l|}{ (P15) Gomes, 2018} \\
\hline (P16) Saraiva, 2018 & & & & $\mathrm{X}$ & $\mathrm{X}$ \\
\hline (P17) Simba, 2017 & $\mathrm{X}$ & & & & $\mathrm{X}$ \\
\hline (P18) Gervásio, 2019 & & & & & $\mathrm{X}$ \\
\hline \multicolumn{6}{|l|}{ (P19) Gomes, 2017} \\
\hline (P20) Pombo, 2019 & & & & & $\mathrm{X}$ \\
\hline (P21) Ferreira, 2015 & & $\mathrm{X}$ & & & $\mathrm{X}$ \\
\hline \multicolumn{6}{|l|}{ (P22) Alexandre, 2020} \\
\hline (P23) Sobreiro, 2017 & $\mathrm{X}$ & & $\mathrm{X}$ & & \\
\hline (P24) Caixado, 2019 & & $\mathrm{X}$ & & & \\
\hline \multicolumn{6}{|l|}{ (P25) Quaresma, 2019} \\
\hline (P26) Coelho, 2017 & & $\mathrm{X}$ & & & $\mathrm{X}$ \\
\hline Total $(\%)$ & $6(23)$ & $5(19)$ & $4(15)$ & $2(8)$ & $11(42)$ \\
\hline
\end{tabular}

Fonte: Elaborado pelos autores, 2021.

\subsection{Caracterização das experiências de gamificação}

Para caracterização das experiências de gamificação, como referido anteriormente, tomamos em consideração todas as referências explícitas dos autores sobre os princípios considerados na configuração das propostas pedagógicas respectivas, mas também as referências feitas aos elementos do jogo considerados e, por fim, às tecnologias mobilizadas para a concretização dessas mesmas experiências de ensino e aprendizagem gamificadas.

No que se refere ao enquadramento teórico e conceitual tomado como referência, os resultados apresentados no Quadro 5 (Princípios e abordagens teóricas) permitem concluir sobre a predominância de estudos que têm como base as questões relacionadas à motivação dos estudantes (dezesseis estudos). Em segundo lugar, surgem os estudos que tomam como referência a promoção de uma aprendizagem significativa na criação de experiências de aprendizagem gamificadas (cinco estudos). 
Quadro 5 - Princípios e abordagens teóricas

\begin{tabular}{|c|c|c|c|c|c|c|c|}
\hline \multirow[b]{2}{*}{ Autor } & \multicolumn{7}{|c|}{ Princípios e abordagens } \\
\hline & $\begin{array}{l}\text { Aprendiz } \\
\text { agem } \\
\text { significat } \\
\text { iva }\end{array}$ & $\begin{array}{l}\text { Aprendiza } \\
\text { gem } \\
\text { Baseada } \\
\text { em Projeto }\end{array}$ & $\begin{array}{c}\text { Socioco } \\
\text { nstrutivi } \\
\text { smo }\end{array}$ & $\begin{array}{c}\text { Teoria } \\
\text { das } \\
\text { Situações } \\
\text { Didáticas }\end{array}$ & $\begin{array}{c}\text { Conecti } \\
\text { vismo }\end{array}$ & $\begin{array}{c}\text { Teoria } \\
\text { da } \\
\text { Motivaç } \\
\text { ão }\end{array}$ & $\begin{array}{c}\text { Teoria } \\
\text { dos } \\
\text { Estilos } \\
\text { de } \\
\text { Aprendi } \\
\text { zagem }\end{array}$ \\
\hline (P1) Santos, 2018 & & & & & & $\mathrm{X}$ & \\
\hline (P2) Quadros, 2016 & & & & & & $\mathrm{X}$ & \\
\hline (P3) França, 2016 & & $\mathrm{X}$ & & & & & \\
\hline (P4) Sataka, 2019 & $\mathrm{X}$ & & & & & & \\
\hline (P5) Corcini, 2016 & & & & & & $\mathrm{X}$ & \\
\hline (P6) Jacobsen, 2018 & & & & & & $\mathrm{X}$ & \\
\hline (P7) Rodrigues, 2018 & & & & & & $\mathrm{X}$ & \\
\hline (P8) Crespo, 2019 & & & & & & $\mathrm{X}$ & \\
\hline (P9) Guedes, 2017 & & & & & & $\mathrm{X}$ & \\
\hline (P10) Duarte, 2017 & & & & & & $\mathrm{X}$ & \\
\hline (P11) Pessi, 2018 & $\mathrm{X}$ & & & & & & \\
\hline (P12) Tristão, 2018 & & & & & & $\mathrm{X}$ & \\
\hline (P13) Gomes, 2017 & & & & $\mathrm{X}$ & & & \\
\hline (P14) Silva, 2019 & & & & & & $\mathrm{X}$ & \\
\hline (P15) Gomes, 2018 & & & & & & $\mathrm{X}$ & \\
\hline (P16) Saraiva, 2018 & & & & & $\mathrm{X}$ & & \\
\hline (P17) Simba, 2017 & & & & & & $\mathrm{X}$ & \\
\hline (P18) Gervásio, 2019 & $\mathrm{X}$ & & & & & & \\
\hline (P19) Gomes, 2017 & $\mathrm{X}$ & & & & & & \\
\hline (P20) Pombo, 2019 & & & $\mathrm{X}$ & & & & \\
\hline (P21) Ferreira, 2015 & & & & & & & $\mathrm{X}$ \\
\hline (P22) Alexandre, 2020 & & & & & & $\mathrm{X}$ & \\
\hline (P23) Sobreiro, 2017 & & & & & & $\mathrm{X}$ & \\
\hline (P24) Caixado, 2019 & & & & & & $\mathrm{X}$ & \\
\hline (P25) Quaresma, 2019 & $\mathrm{X}$ & & & & & & \\
\hline (P26) Coelho, 2017 & & & & & & $\mathrm{X}$ & \\
\hline Total $(\%)$ & $5(19)$ & $1(4)$ & $1(4)$ & $1(4)$ & $1(4)$ & $16(61)$ & $1(4)$ \\
\hline
\end{tabular}

Fonte: Elaborado pelos autores, 2021.

Como exemplo do recurso à teoria da motivação, destacamos a investigação de Tristão (2018), que buscou analisar a influência da gamificação na motivação e no engajamento dos discentes, bem como identificar os aspectos positivos e negativos sob a perspectiva do docente, e propor um modelo de gamificação fazendo uso de atribuição de badges e de uma barra de progresso enquanto fatores motivadores.

No que se refere aos elementos do jogo mobilizados para configuração das experiências de aprendizagem gamificadas, no Quadro 6 (Elementos do jogo) é possível observar a grande riqueza e variedade de recursos utilizados no conjunto dos 26 estudos. Por outro lado, é também possível constatar que a quase totalidade das experiências gamificadas é constituída por cinco ou mais elementos. Na procura de um eventual padrão dominante, destaca-se a atribuição de pontos (em 65\% dos estudos), a disponibilização de feedback (58\%), o estabelecimento de rankings (50\%) e níveis (42\%). Logo a seguir surgem os desafios e a atribuição de badges, em $31 \%$ dos estudos, tendo menor expressão os restantes elementos do jogo mencionados, com percentagens abaixo dos $30 \%$. 
Quadro 6 - Elementos do jogo

\begin{tabular}{|c|c|c|c|c|c|c|c|c|c|c|c|c|c|c|c|c|}
\hline \multirow[b]{2}{*}{ Autor } & \multicolumn{16}{|c|}{ Elementos do jogo } \\
\hline & 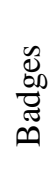 & 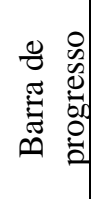 & 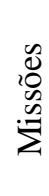 & 串 & 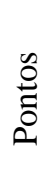 & $\frac{n}{2}$ & 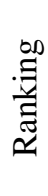 & 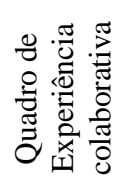 & 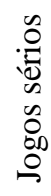 & $\begin{array}{l}0 \\
0 \\
0 \\
0 \\
0 \\
\frac{0}{2} \\
>\end{array}$ & 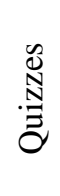 & 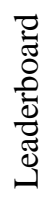 & 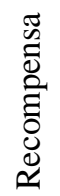 & 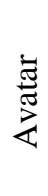 & 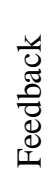 & 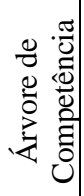 \\
\hline $\begin{array}{l}\text { (P1) Santos, } \\
2018\end{array}$ & & & & & & & & & & & & & & & & \\
\hline $\begin{array}{l}\text { (P2) Quadros, } \\
2016\end{array}$ & & $\mathrm{X}$ & & $\mathrm{X}$ & $X$ & $\mathrm{X}$ & $\mathrm{X}$ & & & & & & $\mathrm{X}$ & & $\mathrm{X}$ & \\
\hline $\begin{array}{l}\text { (P3) França, } \\
2016\end{array}$ & & & & & $\mathrm{X}$ & $\mathrm{X}$ & $\mathrm{X}$ & $\mathrm{X}$ & & & & & & & & \\
\hline $\begin{array}{l}\text { (P4) Sataka, } \\
2019\end{array}$ & & & & $\mathrm{X}$ & & $\mathrm{X}$ & $\mathrm{X}$ & & & & & & $\mathrm{X}$ & & $\mathrm{X}$ & \\
\hline $\begin{array}{l}\text { (P5) Corcini, } \\
2016\end{array}$ & & $\mathrm{X}$ & $\mathrm{X}$ & $X$ & $X$ & & $X$ & & & & & & $\mathrm{X}$ & $\mathrm{X}$ & & \\
\hline $\begin{array}{l}\text { (P6) Jacobsen, } \\
2018\end{array}$ & & & $\mathrm{X}$ & $\mathrm{X}$ & $\mathrm{X}$ & $\mathrm{X}$ & & & & & & & $\mathrm{X}$ & & $\mathrm{X}$ & \\
\hline $\begin{array}{l}\text { (P7) } \\
\text { Rodrigues, } \\
2018 \\
\end{array}$ & $\mathrm{X}$ & & $\mathrm{X}$ & $\mathrm{X}$ & $\mathrm{X}$ & $\mathrm{X}$ & $\mathrm{X}$ & & & & & & $\mathrm{X}$ & & & \\
\hline $\begin{array}{l}\text { (P8) Crespo, } \\
2019\end{array}$ & & & & & & & & & & & & & & & & \\
\hline $\begin{array}{l}\text { (P9) Guedes, } \\
2017\end{array}$ & & & $\mathrm{X}$ & $\mathrm{X}$ & $\mathrm{X}$ & $\mathrm{X}$ & & & $\mathrm{X}$ & & & & & & $\mathrm{X}$ & \\
\hline $\begin{array}{l}\text { (P10) Duarte, } \\
2017\end{array}$ & & & & & & & & & & $\mathrm{X}$ & & & & & $\mathrm{X}$ & \\
\hline $\begin{array}{l}\text { (P11) Pessi, } \\
2018\end{array}$ & & & & & $\mathrm{X}$ & & $\mathrm{X}$ & & & & & & $\mathrm{X}$ & & & \\
\hline $\begin{array}{l}\text { (P12) Tristão, } \\
2018\end{array}$ & $\mathrm{X}$ & $\mathrm{X}$ & & & & & & & & & & & & & $\mathrm{X}$ & \\
\hline $\begin{array}{l}\text { (P13) Gomes, } \\
2017\end{array}$ & & & & & & & & & & & & & & & & \\
\hline $\begin{array}{l}\text { (P14) Silva, } \\
2019\end{array}$ & & & & & $X$ & & $\mathrm{X}$ & & $\mathrm{X}$ & & & & & & $\mathrm{X}$ & \\
\hline $\begin{array}{l}\text { (P15) Gomes, } \\
2018\end{array}$ & $\mathrm{X}$ & & & $\mathrm{X}$ & $\mathrm{X}$ & & $\mathrm{X}$ & & & $\mathrm{X}$ & $\mathrm{X}$ & $\mathrm{X}$ & & & $\mathrm{X}$ & \\
\hline $\begin{array}{l}\text { (P16) Saraiva, } \\
2018\end{array}$ & & & & & & & $\mathrm{X}$ & & & & & & $\mathrm{X}$ & $\mathrm{X}$ & & \\
\hline $\begin{array}{l}\text { (P17) Simba, } \\
2017\end{array}$ & & & $\mathrm{X}$ & & $\mathrm{X}$ & $\mathrm{X}$ & & & & & & & $\mathrm{X}$ & & $\mathrm{X}$ & \\
\hline $\begin{array}{l}\text { (P18) } \\
\text { Gervásio, } 2019\end{array}$ & & & & & $\mathrm{X}$ & $\mathrm{X}$ & & & & $\mathrm{X}$ & $\mathrm{X}$ & & & & & \\
\hline $\begin{array}{l}\text { (P19) Gomes, } \\
2017\end{array}$ & $\mathrm{X}$ & & & & $\mathrm{X}$ & & $\mathrm{X}$ & & & $\mathrm{X}$ & & $\mathrm{X}$ & $\mathrm{X}$ & $\mathrm{X}$ & $\mathrm{X}$ & \\
\hline $\begin{array}{l}\text { (P20) Pombo, } \\
2019\end{array}$ & $\mathrm{X}$ & & & & & & & & & & $\mathrm{X}$ & & & $\mathrm{X}$ & $\mathrm{X}$ & \\
\hline $\begin{array}{l}\text { (P21) Ferreira, } \\
2015\end{array}$ & & & & & $\mathrm{X}$ & $\mathrm{X}$ & $\mathrm{X}$ & & & & & & & & $\mathrm{X}$ & \\
\hline $\begin{array}{l}\text { (P22) } \\
\text { Alexandre, } \\
2020\end{array}$ & & & & & $\mathrm{X}$ & & & & & & & & & $\mathrm{X}$ & $\mathrm{X}$ & $\mathrm{X}$ \\
\hline $\begin{array}{l}\text { (P23) Sobreiro, } \\
2017\end{array}$ & $X$ & $\mathrm{X}$ & & $\mathrm{X}$ & $X$ & $\mathrm{X}$ & $\mathrm{X}$ & & & & & & $\mathrm{X}$ & & $X$ & \\
\hline $\begin{array}{l}\text { (P24) Caixado, } \\
2019\end{array}$ & $\mathrm{X}$ & & & & $\mathrm{X}$ & & & & & & & & & $\mathrm{X}$ & & \\
\hline $\begin{array}{l}\text { (P25) } \\
\text { Quaresma, } \\
2019\end{array}$ & & & & & $\mathrm{X}$ & $\mathrm{X}$ & & & & & & & $\mathrm{X}$ & $\mathrm{X}$ & $\mathrm{X}$ & \\
\hline
\end{tabular}


Revista de Educação, Ciência e Tecnologia

\begin{tabular}{|c|c|c|c|c|c|c|c|c|c|c|c|c|c|c|c|c|}
\hline $\begin{array}{l}\text { (P26) Coelho, } \\
2017\end{array}$ & $\mathrm{X}$ & $\mathrm{x}$ & & & & & $\mathrm{X}$ & & & $\mathrm{x}$ & & & & & & \\
\hline Total $(\%)$ & $\underset{\infty}{\widehat{\vec{c}}}$ & $\underset{n}{\stackrel{\overbrace{}}{\rightleftarrows}}$ & $\underset{n}{\stackrel{\overbrace{}}{\rightleftarrows}}$ & $\underset{\infty}{\stackrel{్}{\sigma}}$ & $\begin{array}{l}\sqrt{\hat{\sigma}} \\
\underline{z}\end{array}$ & $\begin{array}{l}\text { Iิ } \\
=\end{array}$ & $\begin{array}{l}\hat{B} \\
\stackrel{6}{0} \\
\underline{m}\end{array}$ & 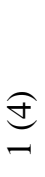 & $\underset{\sim}{\infty}$ & $\underset{n}{\stackrel{\overbrace{}}{\rightleftharpoons}}$ & $\underset{m}{\Xi}$ & $\underbrace{\infty}_{N}$ & $\begin{array}{l}\underset{\mathcal{J}}{\Xi} \\
=\end{array}$ & $\underset{\sim}{\widehat{d}}$ & $\begin{array}{l}\infty \\
\infty \\
n \\
n\end{array}$ & $\underset{-}{\mathbb{E}}$ \\
\hline
\end{tabular}

Fonte: Elaborado pelos autores, 2021.

O estudo de Gomes (2018), com o objetivo de criar um desenho gamificado diferenciador no ensino superior online, pode ser um exemplo elucidativo da conjugação de diferentes elementos do jogo. A estratégia de gamificação criada, neste caso, inclui: uma narrativa, vídeos de apoio à narrativa, quizzes, pontos, badges, leaderboard, avatar, desafios, e feedback de diversa ordem, como feedback da equipe do projeto aos estudantes e feedback entre pares.

No que se refere ao uso de tecnologias digitais (ver Quadro 7. Tecnologias utilizadas), os estudos selecionados mostram que todos os investigadores recorreram a algum tipo de tecnologia na configuração das experiências de gamificação. É, aliás, visível, também neste caso, uma grande diversidade de ferramentas, entre plataformas, aplicações e outro software, todas elas com o propósito de permitir a concretização dos diferentes componentes do jogo.

Quadro 7 - Tecnologias utilizadas

\begin{tabular}{|c|c|c|c|c|c|c|c|c|c|c|c|c|c|c|c|c|c|}
\hline \multirow[b]{2}{*}{ Autor } & \multicolumn{17}{|c|}{ Plataformas, aplicações, software } \\
\hline & 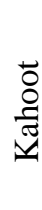 & $\stackrel{N}{*}$ & $\frac{7}{3}$ & $\begin{array}{l}8 \\
\&\end{array}$ & : & $\frac{b}{m}$ & 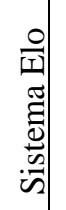 & $\begin{array}{l}\frac{0}{0} \\
0 \\
0 \\
\Sigma\end{array}$ & 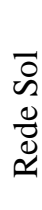 & 总 & $\begin{array}{l}y \\
0 \\
0 \\
0 \\
0 \\
0\end{array}$ & $\frac{60}{\infty I}$ & 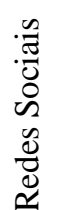 & $\underset{\mho}{\mathbb{\sigma}}$ & 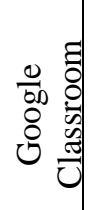 & 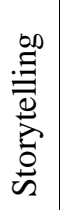 & 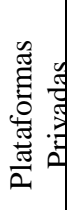 \\
\hline $\begin{array}{l}\text { (P1) Santos, } \\
2018\end{array}$ & & & & & & & & $\mathrm{X}$ & & & & & & & & & \\
\hline $\begin{array}{l}\text { (P2) Quadros, } \\
2016\end{array}$ & & $\mathrm{X}$ & & & & & $\mathrm{X}$ & & & & & & & & & & \\
\hline $\begin{array}{l}\text { (P3) França, } \\
2016\end{array}$ & & & & & & & & $\mathrm{X}$ & & & & & & & & & \\
\hline $\begin{array}{l}\text { (P4) Sataka, } \\
2019\end{array}$ & & & & & $\mathrm{X}$ & & & & & & & & & & & & \\
\hline $\begin{array}{l}\text { (P5) Corcini, } \\
2016\end{array}$ & & & & & & & & & & $\mathrm{X}$ & & & & & & & \\
\hline $\begin{array}{l}\text { (P6) Jacobsen, } \\
2018\end{array}$ & & & & & & & & $\mathrm{X}$ & & & & & & & & & \\
\hline $\begin{array}{l}\text { (P7) Rodrigues, } \\
2018\end{array}$ & $\mathrm{X}$ & & & & & & & & & & & & & & & & \\
\hline $\begin{array}{l}\text { (P8) Crespo, } \\
2019\end{array}$ & $\mathrm{X}$ & & & & & & & & & & & & & & & & \\
\hline $\begin{array}{l}\text { (P9) Guedes, } \\
2017\end{array}$ & & & & & & & & & & & & & $\mathrm{X}$ & & & $\mathrm{X}$ & \\
\hline $\begin{array}{l}\text { (P10) Duarte, } \\
2017\end{array}$ & & & & & & & $\mathrm{X}$ & & & & & & & & & & \\
\hline $\begin{array}{l}\text { (P11) Pessi, } \\
2018\end{array}$ & $X$ & & & & & & & & & & & & & & $\mathrm{X}$ & & \\
\hline $\begin{array}{l}\text { (P12) Tristão, } \\
2018\end{array}$ & & & & & & & & $\mathrm{X}$ & & & & & & & & & \\
\hline $\begin{array}{l}\text { (P13) Gomes, } \\
2017\end{array}$ & & & & & & & & & & & & & & & & & \\
\hline
\end{tabular}




\begin{tabular}{|c|c|c|c|c|c|c|c|c|c|c|c|c|c|c|c|c|c|}
\hline $\begin{array}{l}\text { (P14) Silva, } \\
2019\end{array}$ & & & & & & & & & & & & & & & & & $\mathrm{X}$ \\
\hline $\begin{array}{l}\text { (P15) Gomes, } \\
2018\end{array}$ & & & & & & & & $\mathrm{X}$ & $\mathrm{X}$ & & & & & & & & \\
\hline $\begin{array}{l}\text { (P16) Saraiva, } \\
2018\end{array}$ & & & & & & & & & $\mathrm{X}$ & & & $\mathrm{X}$ & & & & & \\
\hline $\begin{array}{l}\text { (P17) Simba, } \\
2017\end{array}$ & & & $\mathrm{X}$ & & & & & & & & & & & & & & \\
\hline $\begin{array}{l}\text { (P18) Gervásio, } \\
2019\end{array}$ & & & & & & & & $\mathrm{X}$ & & & & & & & & & \\
\hline $\begin{array}{l}\text { (P19) Gomes, } \\
2017\end{array}$ & & & & & & & & $X$ & & & & & & & & & \\
\hline $\begin{array}{l}\text { (P20) Pombo, } \\
2019\end{array}$ & & $\mathrm{X}$ & $\mathrm{X}$ & & & & & $\mathrm{X}$ & & & & & $\mathrm{X}$ & & & & \\
\hline $\begin{array}{l}\text { (P21) Ferreira, } \\
2015\end{array}$ & & & & & & & & & & & $X$ & & $\mathrm{X}$ & $\mathrm{X}$ & & & \\
\hline $\begin{array}{l}\text { (P22) } \\
\text { Alexandre, } \\
2020\end{array}$ & & & & & & $\mathrm{X}$ & & & & & & & & & & & \\
\hline $\begin{array}{l}\text { (P23) Sobreiro, } \\
2017\end{array}$ & & $\mathrm{X}$ & & & & $\mathrm{X}$ & & & & & & & & & & & \\
\hline $\begin{array}{l}\text { (P24) Caixado, } \\
2019\end{array}$ & & & & & & & & & & & & & $\mathrm{X}$ & & & & \\
\hline $\begin{array}{l}\text { (P25) } \\
\text { Quaresma, } 2019\end{array}$ & & & & & & & & $\mathrm{X}$ & & & & & & & & & \\
\hline $\begin{array}{l}\text { (P26) Coelho, } \\
2017\end{array}$ & & & & $\mathrm{X}$ & & & & $\mathrm{X}$ & & & & & & & & & \\
\hline Total (\%) & $\underset{m}{\exists}$ & $\underset{m}{\Xi}$ & $\underbrace{\infty}_{\sim}$ & $\underset{\beth}{\overparen{J}}$ & $\underset{\smile}{\overparen{\Xi}}$ & $\overbrace{\infty}^{\infty}$ & $\begin{array}{l}\infty \\
\text { N }\end{array}$ & $\begin{array}{l}\infty \\
\stackrel{\infty}{0} \\
0 \\
0\end{array}$ & $\overbrace{\infty}^{\infty}$ & $\underset{\exists}{Ð}$ & $\mathcal{Ð}$ & $\underset{\smile}{\overparen{\Xi}}$ & $\underset{\forall}{\stackrel{(2}{=}}$ & $\underset{乙}{\overparen{\Xi}}$ & $\stackrel{Ð}{\mathcal{J}}$ & $\stackrel{Ð}{\Xi}$ & $\underset{Ð}{\overparen{f}}$ \\
\hline
\end{tabular}

Fonte: Elaborado pelos autores, 2021.

A plataforma Moodle, utilizada como base para a gamificação em dez estudos (38\%), destaca-se como sendo o recurso mais utilizado nos estudos aqui considerados. Observa-se, por outro lado, que apenas em um dos estudos utiliza uma plataforma que foi especialmente concebida com o propósito de promover, em particular, o ensino de línguas estrangeiras (Duolingo). A maior parte das restantes ferramentas digitais, principalmente ferramentas de acesso aberto e não sendo propriamente desenhadas com o objetivo de gamificação da aprendizagem, acaba por ser adaptada para esse fim, individualmente ou em combinações, conforme os casos. Como exemplo de uso de uma dessas aplicações, referimos ao estudo de Crespo (2019) que analisa aulas de Alemão em um curso de Letras, nas quais o Kahoot! foi empregado com o objetivo de proporcionar atividades envolvendo a dimensão lúdica na sua configuração.

\subsection{Principais metodologias utilizadas nas pesquisas}

De acordo com o Quadro 8 (Metodologias de investigação), no que se refere às escolhas dos autores selecionados em relação ao tipo de metodologia científica adotada para a realização dos seus estudos, podemos observar que há uma predominância dos estudos de carácter qualitativo relativamente aos estudos quantitativos, com um total de quinze (58\%) no primeiro caso e de apenas dois (8\%) no segundo caso, muito embora seis deles recorrem a metodologias mistas. 
Quadro 8 - Metodologias de investigação

\begin{tabular}{|c|c|c|c|c|}
\hline & \multicolumn{4}{|c|}{ Natureza da investigação } \\
\hline & Quantitativa & Qualitativa & Mista & DBR \\
\hline (P1) Santos, 2018 & & $\mathrm{X}$ & & \\
\hline (P2) Quadros, 2016 & & & $\mathrm{X}$ & \\
\hline (P3) França, 2016 & & & $\mathrm{X}$ & \\
\hline (P4) Sataka, 2019 & & $\mathrm{X}$ & & \\
\hline (P5) Corcini, 2016 & & $\mathrm{X}$ & & \\
\hline (P6) Jacobsen, 2018 & & $\mathrm{X}$ & & \\
\hline (P7) Rodrigues, 2018 & & $\mathrm{X}$ & & \\
\hline (P8) Crespo, 2019 & & & $\mathrm{X}$ & \\
\hline (P9) Guedes, 2017 & & & $\mathrm{X}$ & \\
\hline (P10) Duarte, 2017 & & $\mathrm{X}$ & & \\
\hline (P11) Pessi, 2018 & & $\mathrm{X}$ & & \\
\hline (P12) Tristão, 2018 & & $\mathrm{X}$ & & \\
\hline (P13) Gomes, 2017 & & $\mathrm{X}$ & & \\
\hline (P14) Silva, 2019 & $\mathrm{X}$ & & & \\
\hline (P15) Gomes, 2018 & & & & $\mathrm{X}$ \\
\hline (P16) Saraiva, 2018 & & & & $\mathrm{X}$ \\
\hline (P17) Simba, 2017 & & & $\mathrm{X}$ & \\
\hline (P18) Gervásio, 2019 & & $\mathrm{X}$ & & \\
\hline (P19) Gomes, 2017 & & $\mathrm{X}$ & & \\
\hline (P20) Pombo, 2019 & & & & $\mathrm{X}$ \\
\hline (P21) Ferreira, 2015 & & $\mathrm{X}$ & & \\
\hline (P22) Alexandre, 2020 & & $\mathrm{X}$ & & \\
\hline (P23) Sobreiro, 2017 & & $\mathrm{X}$ & & \\
\hline (P24) Caixado, 2019 & $\mathrm{X}$ & & & \\
\hline (P25) Quaresma, 2019 & & & $\mathrm{X}$ & \\
\hline (P26) Coelho, 2017 & & $\mathrm{X}$ & & \\
\hline Total $(\%)$ & $2(8)$ & $15(58)$ & $6(23)$ & $3(11)$ \\
\hline
\end{tabular}

Fonte: Elaborado pelos autores, 2021.

Referir, por outro lado, que apenas três dos estudos analisados optaram por uma abordagem de metodologia de desenvolvimento (Design Based Research - DBR), uma metodologia que vem sendo cada vez mais usada nas investigações em Educação, nomeadamente quando, como no caso do desenvolvimento de estratégias de gamificação, o objetivo é precisamente o desenho, a implementação e a avaliação de novas propostas de ensino e aprendizagem. É o caso do estudo de Saraiva (2018) em que o autor justifica o uso dessa metodologia precisamente por se tratar da primeira vez que iria ser implementada a Gamificação, tendo por base o recurso a uma rede social acadêmica já existente numa determinada Universidade Virtual. A recolha de dados foi efetuada através de entrevistas semiestruturadas, tendo por objetivo contar com o contributo dos estudantes, tanto para a construção de uma nova rede (SOL2), como para a sua avaliação após a implementação, neste caso por meio da aplicação de questionários. Referente ao estudo de Pombo (2019), a utilização da metodologia de desenvolvimento foi justificada na medida em que tal abordagem permite que o próprio desenvolvimento se apoie em determinados "princípios de desenho", que podem guiar, informar e otimizar as experiências de gamificação a implementar. 
3.4 Principais resultados dos estudos

No intuito de compreender quais os principais resultados obtidos nos estudos selecionados com recurso das experiências de gamificação no ensino superior, a análise de conteúdo centrou-se na identificação dos principais ganhos e vantagens apontados pelos autores, bem como as referências feitas a eventuais dificuldades e limitações verificadas. Conforme se observou no Quadro 9 (Resultados dos estudos), em termos de ganhos e vantagens, o conjunto dos estudos faz referência de forma substantiva e com grande destaque à inovação pedagógica que as experiências acabaram por constituir (em $81 \%$ dos estudos), rompendo abertamente com os métodos tradicionais de ensino, que teria sido, aliás, o principal propósito comum da implementação das diferentes experiências de gamificação. No geral, a gamificação é comumente referida como uma estratégia ou prática pedagógica associada a um aumento da motivação do aluno, a uma maior aproximação e interação entre os discentes e aumento no seu envolvimento. É o que, a título de exemplo, verificou-se no trabalho de Tristão (2018, p. 10) ao mencionar que "A partir dos resultados obtidos na análise dos dados, foi possível identificar que: a barra de progresso motivou mais do que os badges, e, comparativamente à turma anterior, houve aumento de acessos ao ambiente do curso e os perfis dos participantes foram muito mais visualizados. Sob a perspectiva do tutor, a gamificação aumentou o envolvimento dos alunos e a qualidade dos trabalhos desenvolvidos".

Embora em todos os estudos haja apontamentos às vantagens que esse tipo de experiência pode trazer ao processo de ensino e aprendizagem, apenas cinco estudos (19\%) fazem referências mais explícitas aos ganhos efetivos em termos de aprendizagem com recurso às experiências de gamificação levadas a cabo. É o que parece sugerir Gervásio (2019, p. 67), quando afirma que "esses recursos favorecem o ensino de línguas de modo mais dinâmico e interativo, além de promover a aprendizagem significativa, já que os plug-ins permitem criar atividades que explorem elementos multimodais, tornando o processo de aprendizagem mais ativo".

Quadro 9 - Resultados dos estudos

\begin{tabular}{|l|c|c|c|}
\hline \multirow{2}{*}{\multicolumn{1}{|c|}{ Autor }} & \multicolumn{3}{c|}{ Resultados } \\
\cline { 2 - 4 } & $\begin{array}{c}\text { Ganhos / vantagens } \\
\text { inovação } \\
\text { pedagógica }\end{array}$ & $\begin{array}{c}\text { aprendizagem } \\
\text { dos alunos }\end{array}$ & \\
\hline (P1) Santos, 2018 & $\mathrm{X}$ & & \\
\hline (P2) Quadros, 2016 & & $\mathrm{X}$ & \\
\hline (P3) França, 2016 & $\mathrm{X}$ & & \\
\hline (P4) Sataka, 2019 & $\mathrm{X}$ & & $\mathrm{X}$ \\
\hline (P5) Corcini, 2016 & $\mathrm{X}$ & & $\mathrm{X}$ \\
\hline (P6) Jacobsen, 2018 & $\mathrm{X}$ & & $\mathrm{X}$ \\
\hline (P7) Rodrigues, 2018 & $\mathrm{X}$ & & \\
\hline (P8) Crespo, 2019 & $\mathrm{X}$ & & $\mathrm{X}$ \\
\hline (P9) Guedes, 2017 & $\mathrm{X}$ & & \\
\hline (P10) Duarte, 2017 & $\mathrm{X}$ & & $\mathrm{X}$ \\
\hline (P11) Pessi, 2018 & $\mathrm{X}$ & & $\mathrm{X}$ \\
\hline (P12) Tristão, 2018 & $\mathrm{X}$ & & \\
\hline (P13) Gomes, 2017 & & & \\
\hline (P14) Silva, 2019 & $\mathrm{X}$ & & $\mathrm{X}$ \\
\hline (P15) Gomes, 2018 & $\mathrm{X}$ & & \\
\hline (P16) Saraiva, 2018 & $\mathrm{X}$ & & \\
\hline (P17) Simba, 2017 & $\mathrm{X}$ & & \\
\hline (P18) Gervásio, 2019 & & & \\
\hline
\end{tabular}




\begin{tabular}{|l|c|c|c|}
\hline (P19) Gomes, 2017 & X & & $X$ \\
\hline (P20) Pombo, 2019 & X & & \\
\hline (P21) Ferreira, 2015 & & $X$ & \\
\hline (P22) Alexandre, 2020 & & $X$ & \\
\hline (P23) Sobreiro, 2017 & X & & \\
\hline (P24) Caixado, 2019 & X & & \\
\hline (P25) Quaresma, 2019 & X & & \\
\hline (P26) Coelho, 2017 & $\mathrm{X}$ & & $7(27)$ \\
\hline Total $(\%)$ & $21(81)$ & $5(19)$ & \\
\hline
\end{tabular}

Fonte: Elaborado pelos autores, 2021.

Embora na totalidade dos estudos analisados, os respectivos autores se mostrem globalmente satisfeitos com as experiências de gamificação implementadas, importa referir aqui algumas das limitações ou desvantagens apontadas por alguns dos autores, tais como as questões relacionadas com as novas exigências que a gamificação vem trazer, por exemplo, a nível da gestão do tempo, da própria organização da avaliação das aprendizagens, ou mesmo do ponto de vista das competências tecnológicas necessárias, quer por parte do professor, quer por parte dos estudantes. Sobre os desafios em termos de avaliação das aprendizagens, é elucidativo o caso de Pessi (2018, p. 121), quando refere que,

Logo no início das aulas, quando apresentado o plano de atividades da disciplina [aos estudantes], os mesmos questionaram se a pontuação obtida teria relação com as notas bimestrais. Diante dessa experiência, optou-se em gamificar a avaliação, o que nos levou a refletir sobre a importância de criar novos modelos avaliativos, que saiam dos padrões formais e configuram-se em instrumentos realmente significativos para a aprendizagem do aluno.

As dificuldades relacionadas aos aspectos tecnológicos são mencionadas, por exemplo, no estudo de Rodrigues (2018, p. 53) quando refere,

[...] a demora na disponibilização do ambiente virtual para que a disciplina pudesse iniciar suas atividades juntamente com as outras presenciais; as limitações apresentadas pelo portal em receber as atividades em formato de vídeo obrigando a docente a disponibilizar seu e-mail pessoal para envio dos trabalhos e dificultando o controle pela pesquisadora quanto ao cumprimento do prazo referente a segunda unidade da disciplina; as oscilações de disponibilidade de conexão à internet obrigando a postergação de atividades e comprometendo a eficácia dos resultados.

\section{Considerações Finais}

Tal como pudemos observar no ponto anterior, a análise dos resultados dos estudos selecionados mostra que, de uma forma ou outra, todas as investigações fazem, em termos gerais, uma avaliação positiva do recurso a estratégias de gamificação do processo de ensino e aprendizagem. As considerações positivas estão associadas principalmente a questões que envolvem a gamificação como inovação pedagógica, capaz de gerar um aumento da motivação, maior interação e aproximação dos estudantes com os docentes e maior envolvimento no trabalho acadêmico. Embora nem todos os trabalhos analisados se referem de forma explícita aos ganhos em termos de aprendizagem propriamente dita, as referências a uma avaliação 
positiva das estratégias de gamificação implementadas, sugerem que de alguma maneira a gamificação pode vir a ter uma implementação efetiva no contexto acadêmico à medida que as experiências vão sendo desenvolvidas e, sobretudo, com a partilha do conhecimento que naturalmente venha a ser feita sobre os processos utilizados e sobre os resultados obtidos.

Muito embora ainda seja uma área de estudo recente, são cada vez mais numerosos os trabalhos acadêmicos que se debruçam sobre experiências de gamificação da aprendizagem implementadas no contexto do ensino superior. Surgindo sistematicamente como forma de reação a níveis elevados de desmotivação dos estudantes face aos modelos tradicionais de ensino baseados em aulas magistrais e na memorização da informação, essas experiências pedagógicas baseadas na implementação de uma dimensão lúdica e de um maior protagonismo do estudante constituem também uma oportunidade interessante para exploração e análise, do ponto de vista investigativo. Em particular, pelo fato de os investigadores que conduzem os estudos e, também, concebem e implementam as experiências de aprendizagem gamificadas que são objeto de análise. Se do ponto de vista da investigação isso levanta desafios novos, nomeadamente em termos de garantia de objetividade da análise, acaba por constituir também uma oportunidade única em termos de reflexão, tanto em termos pedagógicos como em termos de desenvolvimento profissional docente. Trabalho de elevada exigência, é certo, mas que constituirá o caminho a seguir se o objetivo for o de mudar a face sobre como se ensina e organiza a aprendizagem nas instituições de ensino superior num contexto fortemente marcado pelo desenvolvimento tecnológico e pela natural adesão dos jovens aos jogos e às tecnologias digitais que hoje fazem parte do seu cotidiano. A opção que neste trabalho tomamos de focarmos apenas em estudos acadêmicos realizados por investigadores de língua portuguesa, tinha de alguma forma o intuito de percebermos melhor o que nesse contexto tem sido feito, não só porque também sentimos a desmotivação dos estudantes com quem trabalhamos (em Portugal e no Brasil), mas sobretudo porque nos move a procurar soluções que constituam alternativas efetivas, tendo em vista o seu envolvimento ativo no que lhes é proposto em termos de aprendizagem.

\section{Referências}

BAI, S.; HEW, K.; HUANG, B. Does gamification improve student learning outcome? Evidence from a meta-analysis and synthesis of qualitative data in educational contexts. Educational Research Review, v. 30, p. 100322-100322, 2020.

BITTENTUIT Jr., J. Gamificação na Educação: revisão sistemática de estudos empíricos disponíveis na Biblioteca Digital Brasileira de Teses e Dissertações. Revista Temática, v. 16, n. 3, p. 285-301, 2020.

CARVALHO, F. Gestão do conhecimento. São Paulo: Person, 2012.

CSIKSZENTMIHALYI, M. Flow: The Psychology of Optimal Experience. Academy of Management Review, p. 636-640, 1990.

DETERDING, S. et al. Gamification: Toward a Definition. CHI 2011 Gamification Workshop Proceedings, Vancouver, p. 12-15, 2011.

HAMARI, J. Gamification. The Blackwell Encyclopedia of Sociology. Oxford, UK: John Wiley \& Sons Ltd, 2019. p. 1-3. 
HASSAN, M. et al. Adaptive gamification in e-learning based on students' learning styles. Interactive Learning Environments, p. 1-21, 2019.

HUOTARI, K.; HAMARI, J. Defining Gamification: A Service Marketing Perspective. In: Proceeding of the 16th International Academic MindTrek Conference. New York, NY: ACM, 2012. p. 17-22.

IMBELLONI, L. Títulos de trabalhos científicos: obrigado pela informação contida em seu título. Revista Brasileira Anestesiologia, v. 62, n. 2, p. 139-40, 2012.

KUO, M. S.; CHUANG, T. Y. How gamification motivates visits and engagement for online academic dissemination - an empirical study. Computers in Human Behavior, v. 55, p. 1627, 2016.

MAZUR, E. La educación formal acaba con nuestra motivación intrínseca por aprender, 2020. Disponível em:

https://elpais.com/economia/2020/06/03/actualidad/1591148852_706097.html . Acesso em: 28 maio 2021.

OLIVEIRA, W.; BITTENCOURT, I. Tailored Gamification to Educational Technologies. São Paulo: Springer, 2019.

PARREÑO, J.; IBÁÑEZ, E.; ARROYO, A. The use of gamification in education: a bibliometric and text mining analysis. Journal of Computer Assisted Learning, v. 32, p. 6, p. 663-676, 2016.

PRENSKY, M. Aprendizagem baseada em jogos digitais. São Paulo: Senac-SP, 2012.

TENÓRIO, T. et al. A gamified peer assessment model for on-line learning environments in a competitive context, v. 64, p. 247-263, 2016.

VIAMONTE, A. Uma experiência de gamificação no ensino superior. In: COSTA, M. J. (Ed.). Congresso Nacional de Práticas Pedagógicas no Ensino Superior. Braga, 2018. P. 17-23.

Recebido em março de 2021.

Aprovado em maio de 2021. 


\section{APENDICE}

Lista dos estudos analisados

\begin{tabular}{|c|c|c|}
\hline & Autor & Título \\
\hline P1 & Santos, Lidiane Rocha dos & $\begin{array}{l}\text { A cognição inventiva na docência universitária: } \\
\text { das narrativas de si aos relatos de experiências } \\
\text { de práticas pedagógicas gamificadas }\end{array}$ \\
\hline $\mathrm{P} 2$ & $\begin{array}{l}\text { Quadros, Gerson Bruno } \\
\text { Forgiarini de }\end{array}$ & A gamificação no ensino de línguas online \\
\hline P3 & França, Rômulo Martins & $\begin{array}{l}\text { Ambiente gamificado de aprendizagem baseada } \\
\text { em projetos }\end{array}$ \\
\hline $\mathrm{P} 4$ & Sataka, Mayara Mayumi & $\begin{array}{l}\text { Análise do aplicativo Duolingo para } \\
\text { aprendizagem de Língua Espanhola: uma } \\
\text { pesquisa narrativa }\end{array}$ \\
\hline P5 & Corcini, Luiz Fernando & $\begin{array}{l}\text { Cenários imersivos de aprendizagem: a } \\
\text { construção do conhecimento em ambientes de } \\
\text { pós-graduação. }\end{array}$ \\
\hline P6 & Jacobsen, Daniel de Melo & $\begin{array}{l}\text { Contribuições da gamificação para o ensino e a } \\
\text { aprendizagem: uma proposta de ensino para } \\
\text { matemática financeira }\end{array}$ \\
\hline P7 & Rodrigues, Larissa Berredo & $\begin{array}{l}\text { Educação e tecnologias digitais: a gestão } \\
\text { escolar que alia a estratégia da gamificação ao } \\
\text { processo de ensino-aprendizagem }\end{array}$ \\
\hline P8 & Crespo, Arthur Heredia & $\begin{array}{l}\text { Elementos do Estado de Fluxo durante } \\
\text { atividades com Kahoot!: um estudo de casos } \\
\text { múltiplos em aulas de alemão }\end{array}$ \\
\hline P9 & Guedes, Anibal Lopes & $\begin{array}{l}\text { Emancipação digital cidadã de jovens do } \\
\text { campo num contexto híbrido, multimodal e } \\
\text { ubíquo }\end{array}$ \\
\hline P10 & Duarte, Gabriela Bohlmann & $\begin{array}{l}\text { Eventos complexos de letramentos na } \\
\text { aprendizagem de inglês: relações entre práticas } \\
\text { de letramentos, gamificação e motivação }\end{array}$ \\
\hline P11 & Pessi, Ingrid Gayer & $\begin{array}{l}\text { Gamificação como estratégia pedagógica na } \\
\text { formação do pedagogo }\end{array}$ \\
\hline P12 & Tristão, Patrícia da Silva & $\begin{array}{l}\text { Gamificação da disciplina metodologia da } \\
\text { pesquisa no ensino superior: estudo de caso }\end{array}$ \\
\hline P13 & Gomes, Marcelo dos Santos & $\begin{array}{l}\text { Gamificação e Educação Matemática: uma } \\
\text { reflexão pela óptica da teoria das situações } \\
\text { didáticas }\end{array}$ \\
\hline $\mathrm{P} 14$ & Silva, Rui Jorge Rodrigues & Gamificação no Ensino da Gestão \\
\hline $\mathrm{P} 15$ & Gomes, Cláudia & Gamificação no ensino superior online \\
\hline P16 & $\begin{array}{l}\text { Saraiva, Fernando José Vaz } \\
\text { Guedes Bacelar }\end{array}$ & $\begin{array}{l}\text { Gamificação numa rede social duma } \\
\text { universidade virtual: o caso da Rede SOL }\end{array}$ \\
\hline P17 & Simba Paucar, Sandra Lucia & $\begin{array}{l}\text { Gamificación como estrategia de motivación en } \\
\text { la plataforma virtual de la Educación Superior } \\
\text { Presencial }\end{array}$ \\
\hline P18 & $\begin{array}{l}\text { Gervásio, João Roberto } \\
\text { Ricalde }\end{array}$ & $\begin{array}{l}\text { Investigação da customização da plataforma } \\
\text { AVA "Moodle”" para uso no ensino de línguas a } \\
\text { distância }\end{array}$ \\
\hline P19 & Gomes, Adilson Fernandes & $\begin{array}{l}\text { Material didático digital, games e gamification: } \\
\text { conexões no design para implementação de } \\
\text { cursos online }\end{array}$ \\
\hline P20 & Pombo, Cândida Perpétua & $\begin{array}{l}\text { Mobile learning e educação em línguas: } \\
\text { contributos para a aprendizagem do inglês no } \\
\text { ensino superior online }\end{array}$ \\
\hline
\end{tabular}


Revista de Educação, Ciência e Tecnologia

\begin{tabular}{|l|l|l|}
\hline P21 & Ferreira, Bruno Santos & $\begin{array}{l}\text { O uso da gamificação como estratégia didática } \\
\text { na capacitação de professores para o uso de } \\
\text { softwares educativos }\end{array}$ \\
\hline P22 & $\begin{array}{l}\text { Alexandre, Gabriel } \\
\text { Guimarães }\end{array}$ & $\begin{array}{l}\text { Práticas letradas de gamificação: estudo do } \\
\text { processo de textualização no ensino superior }\end{array}$ \\
\hline P23 & $\begin{array}{l}\text { Sobreiro, Jason Antonio } \\
\text { Pedroso }\end{array}$ & $\begin{array}{l}\text { Proposta de desenvolvimento de instrumento de } \\
\text { aplicação de atividades gamificadas para } \\
\text { disciplinas do ensino }\end{array}$ \\
\hline P24 & $\begin{array}{l}\text { Caixado, Duarte Manuel } \\
\text { Farinha }\end{array}$ & $\begin{array}{l}\text { The gamification features' effect on the training } \\
\text { effectiveness in organizational context }\end{array}$ \\
\hline P25 & $\begin{array}{l}\text { Quaresma, José Augusto de } \\
\text { Sena }\end{array}$ & $\begin{array}{l}\text { Um framework gamificado para a disciplina } \\
\text { algoritmos ou equivalente }\end{array}$ \\
\hline P26 & $\begin{array}{l}\text { Coelho, Janaina Aparecida } \\
\text { Ponté }\end{array}$ & $\begin{array}{l}\text { Uso de gamificação em cursos online abertos e } \\
\text { massivos para formação continuada de } \\
\text { docentes de matemática }\end{array}$ \\
\hline
\end{tabular}

Fonte: Elaborado pelos autores, 2021. 